\title{
LA PERSISTENCIA DE UNA IDEA: EL NACIONALISMO REVOLUCIONARIO. DEL PRI A LÓPEZ OBRADOR
}

\author{
THE PERSISTENCE OF AN IDEA: \\ REVOLUTIONARY NATIONALISM \\ FROM THE PRI TO LÓPEZ OBRADOR
}

\section{LA PERSISTANCE D'UNE IDÉE: LE NATIONALISME RÉVOLUTIONNAIRE DEPUIS L'ĖRE DU PRI JUSQU'AU GOUVERNEMENT DE LÓPEZ OBRADOR}

\author{
Rogelio Hernández Rodríguez \\ El Colegio de México \\ rhernan@colmex.mx
}

\section{A don Rafael Segovia}

\begin{abstract}
RESUMEN: El presente ensayo se propone mostrar cómo el nacionalismo revolucionario fue durante décadas el fundamento de las instituciones y tareas del sistema posrevolucionario y de la sociedad mexicana. Esas ideas, que se convirtieron en programas de gobierno y en instituciones para aplicarlos, dieron resultados que caracterizaron al Estado y su función, así como al partido dominante, parte integral de este desarrollo institucional. La transformación más profunda de estos principios y del Estado mismo comenzó con la sustitución de la política económica y el cambio consecuente de la elite en el poder, iniciada por el gobierno del presidente Miguel de la Madrid. El nacionalismo revolucionario, no obstante, se mantuvo presente en la crítica a los gobiernos, lo mismo del PAN que del PRI, desde 1988, y constituye el respaldo social que dio el triunfo a Andrés Manuel López Obrador en 2018.

Palabras clave: nacionalismo revolucionario; política económica; modernización económica; Enrique Peña Nieto;

Andrés Manuel López Obrador.
\end{abstract}

ABSTRACT: This essay seeks to show how revolutionary nationalism was for decades the foundation of the institutions and efforts of the post-revolution- 
ary system and of Mexican society. These ideas, which were transformed into government programs and institutions to apply them, produced results that defined the state and its functions, together with the dominant party, as an integral part of this institutional development. The deeper transformation of these principles and of the state itself began with the substitution of economic policy and the resulting change in the elite in power, beginning with the government of President Miguel de la Madrid. However, revolutionary nationalism maintained a presence in the criticism of governments, both PAN and PRI, after 1988, and constitutes the social underpinning that swept Andrés Manuel López Obrador to power in 2018.

Keywords: revolutionary nationalism; economic policy; economic modernization; Enrique Peña Nieto; Andrés Manuel López Obrador.

\section{Traducción de Gonzalo Celorio Morayta}

RÉsumé: Cet essai cherche à montrer comment le nationalisme révolutionnaire a été, le long de plusieurs décennies, le fondement des institutions et des activités du système politique issu de la révolution, mais aussi de celles de la société mexicaine. Cette idéologie, qui a engendré un programme de gouvernement et des mécanismes pour le mettre en pratique, a produit des résultats qui à leur tour ont défini le caractère de l'État et de ses fonctions, y compris le rôle du parti dominant à la base du développement institutionnel. Une transformation profonde des principes et de l'État dans son ensemble a été déclenchée par l'adoption d'une politique économique novatrice, suivie du renouvellement de l'élite au pouvoir, à partir de la présidence de Miguel de la Madrid. Cependant, le nationalisme révolutionnaire a survécu et alimenté les critiques contre plusieurs gouvernements -du PAN et du PRI- depuis 1988, jusqu'à servir d'appui à la victoire d'Andrés Manuel López Obrador en 2018.

Mots clefs: Nationalisme révolutionnaire; politique économique; modernisation économique; Enrique Peña Nieto;

Andrés Manuel López Obrador.

\section{Traducción de BERNARdo Mabire}

Fecha de recepción: junio de 2019

Fecha de aceptación: noviembre de 2019 
¿Quién puede estar en contra del nacionalismo revolucionario? Tal vez los timoratos se asusten de la palabra "revolucionario". Pero ¿quién puede estar contra el nacionalismo?, ¿quién puede no desear una democratización integral de la sociedad?, ¿o una sociedad más igualitaria?

L as palabras anteriores no fueron escritas por Jesús Reyes Heroles, Luis Echeverría, José López Portillo ni, menos aún, por Lázaro Cárdenas, sino por Miguel de la Madrid, ${ }^{1}$ el presidente con el que se inició la modificación más profunda de los principios y acciones que caracterizaron el Estado posrevolucionario, la cual, además de sustituir la política económica, llevó al poder a la élite tecnocrática, racionalista e inexperta en la política. En buena medida por ello, después de tres sexenios, el Partido Revolucionario Institucional (PRI) perdió el poder.

Las palabras de De la Madrid revelan que el nacionalismo revolucionario ha sido un componente inseparable de la visión de los gobernantes y, a juzgar por la crítica social, también de la inmensa mayoría de los mexicanos. Aun con un proyecto político por completo distinto al de los presidentes priistas caracterizados como tradicionales, De la Madrid creyó que su proyecto económico, contrario en objetivos y acciones al de sus predecesores, era igualmente nacionalista y revolucionario. Ese planteamiento, usualmente atribuido al priismo como su ideología, que trataba de explicar (justificar, para muchos) su permanencia, en realidad ha dado sustento a la historia nacional, al Estado posrevolucionario y a las principales instituciones del sistema político. Durante décadas, dio sus principios a los gobiernos priistas, fue la base indiscutible de su ruptura en 1987-1988 y ha alimentado la crítica a los gobiernos de la alternancia, lo mismo del Partido Acción Nacional (PAN) que del PRI, hasta dar la razón, el

${ }^{1}$ Miguel de la Madrid, Cambio de rumbo, México, FCE, 2014, p. 787. 
voto y el respaldo social al triunfo de Andrés Manuel López Obrador en 2018.

El presente ensayo tiene el propósito de mostrar cómo surgió esa explicación ideológica y, sobre todo, cómo fue que se enraizó en el pensamiento de la sociedad mexicana. La explicación no se encuentra en un adoctrinamiento ideológico, sino en que se materializó en instituciones y tareas que fue cumpliendo el sistema posrevolucionario. Ideas que no quedaron solamente en eso, sino que se transformaron en programas, resultados sociales y económicos que definieron siempre al Estado y su función. Por razones históricas, el partido dominante formó parte de ese desarrollo institucional, que se apropió por completo de la herencia revolucionaria y despojó a sus opositores, incluida en primer lugar la izquierda, de discurso y propuestas. Hasta que una nueva élite decidió que la Revolución y su herencia eran inaceptables para la modernización económica.

\section{DE LAS IDEAS A LAS INSTITUCIONES}

La asociación formal y explícita del nacionalismo y lo revolucionario apareció en la Declaración de Principios del PRI desde su fundación, en 1946, pero con diversas paráfrasis ya figuraba en los documentos del Partido Nacional Revolucionario (PNR) y, sobre todo, en el de la Revolución Mexicana (PRM). En estos últimos incluso se vinculó abiertamente a la lucha de clases y se definió el propósito del partido como el de crear un gobierno de trabajadores. Aunque, en realidad, la asociación aparece más en los discursos de los dirigentes priistas y de los gobernantes, en los documentos básicos del partido se habla de una organización que se inspira en los “principios ideológicos de la Revolución”, que son el nacionalismo, las libertades y, sobre todo, la justicia social, a los cuales se añadió en las décadas recientes la democracia.

Pero si bien el nacionalismo revolucionario fue adoptado como ideología del partido, no fueron los priistas los que 
le dieron el cuerpo y la coherencia explicativa que le ha sido característica. La elaboración fue propia de académicos e intelectuales, no siempre asociados al PRI. En la Declaración de Principios revisada en 1947 con motivo de la postulación de Miguel Alemán, se dice que el partido tiene el propósito de representar la lucha "permanente" del pueblo por mejorar sus condiciones económicas en busca de la justicia social. ${ }^{2}$ En los años sesenta se hizo explícito que esos objetivos se lograrían mediante la acción de las instituciones surgidas de la Revolución que, además, recogerían la herencia de la Independencia y la Reforma. ${ }^{3}$

El nacionalismo revolucionario es la suma de acontecimientos históricos y objetivos (que, además, se asumen comunes, si no es que iguales) de los movimientos fundacionales de la nación mexicana. Como bien advirtió Rafael Segovia en un ensayo de 1968, a diferencia de la tradición teórica del nacionalismo, en México la nación no se define por los conceptos clásicos de territorio, pueblo o lengua. Ni siquiera los enfrentamientos con otros países (las invasiones estadounidense y francesa) o la raza determinan la idea de nación. Los primeros se integran a los movimientos sociales (las agresiones extranjeras y el despojo de tierras se insertan en una larga historia del siglo xIx que abarca tanto la Independencia como la Reforma), son episodios de la larga lucha del pueblo, y la raza se asume como una característica de ese mismo pueblo mexicano que, además, fundamenta la lucha contra España, el clero y los conservadores, así como contra el porfiriato, la apropiación de la tierra y la explotación. ${ }^{4}$

2 Partido Revolucionario Institucional, Historia documental del partido de la Revolución, México, ICAP, 1982, t. 5, p. 254.

3 Ibid., t. 7, p. 463.

${ }^{4}$ Rafael Segovia, "El nacionalismo mexicano. Los programas políticos revolucionarios (1929-1964)", en El Colegio de México, Centro de Estudios Internacionales, Lecturas de política mexicana, México, El Colegio de México, 1977, pp. 39-42. El artículo fue publicado originalmente en 1968 en Foro Internacional, 4 (32), pp. 349-359. 
La más clara formulación de este principio se debe a las reflexiones de Vicente Lombardo Toledano. Para él, la nación mexicana es el resultado de las luchas del pueblo por liberarse de la opresión y explotación colonial, conservadora y de los hacendados porfiristas, que se logró en tres movimientos históricos: Independencia, Reforma y Revolución. Estos movimientos no responden a circunstancias específicas o a condicionamientos determinados, sino a una especie de motivo común del pueblo que busca su libertad, autonomía y mejoramiento social. Con toda claridad, Lombardo escribió que "la Revolución Mexicana es una: comenzó en 1810 y todavía no termina". ${ }^{5}$ Con cada acontecimiento se alcanzó un objetivo del pueblo: la identidad contra la Colonia, democracia y libertad en la Reforma y, en especial, la justicia social con la Revolución. Gracias a que en cada etapa se alcanzó una meta, es que "existe la nación mexicana". ${ }^{6}$ Así, pueblo, nación e historia son lo mismo.

Como el protagonista de esta historia es el pueblo y tiene siempre un objetivo común, el nacionalismo revolucionario asume como naturales las contradicciones y, por lo tanto, no reconoce objetivos distintos o diferencias fundamentales. Juan J. Linz, en su clásico texto sobre el autoritarismo, afirmó que este tipo de sistemas carecen de ideología, entendida como una explicación lógica y coherente que justifique la existencia de los regímenes, particularmente los dictatoriales. En realidad, Linz tenía en mente las explicaciones marxistas que dieron sustento a los países socialistas, en especial la antigua Unión Soviética, explicaciones más teóricas y genéricas que particulares de cada país. ${ }^{7}$ En su lugar, los autoritarismos se basan en mentalidades que recogen tradiciones, costum-

5 Vicente Lombardo Toledano, "Definición de la nación mexicana”, en su libro La Revolución Mexicana, 1921-1967, t. I, introducción y selección de documentos por Gastón García Cantú, México, inehrm, 1988, p. 261.

6 Ibid., p. 263.

7 Juan J. Linz, "An Authoritarian Regime: Spain”, en Eric Allardt y Stein Rokkan (eds.), Mass Politics, Nueva York, The Free Press, 1970. 
bres, episodios históricos, etcétera, que aunque entrañan contradicciones obvias, no afectan el discurso porque los gobernantes las emplean a discreción y casuísticamente.

Los académicos le resolvieron el problema lógico al nacionalismo revolucionario eliminando las particularidades, creando un único sujeto y un propósito siempre complementario. Por eso, como muchos lo han señalado, el panteón de héroes nacionales es tan disímbolo, más de una vez incongruente, pero plenamente aceptado por el pueblo, la sociedad o los ciudadanos. Por eso también, es posible colocar en una línea común a Hidalgo, Morelos, Juárez, Madero, Villa, Zapata y Lázaro Cárdenas. Todos, en el momento que la historia les asignó, cumplieron con el mismo propósito libertario y de justicia social. ${ }^{8}$

El planteamiento no se detiene en el plano ideológico. Lo que le ha dado consistencia es que se ha materializado en tareas y, sobre todo, en instituciones que deben cumplirlas. La justicia social (lema que acompañó por décadas al PRI) solamente se alcanzaría por la acción del Estado. Y esta elaboración, de nuevo, no la hicieron los priistas, sino los académicos. Alguien tan alejado del PRI y del régimen, como fue Arnaldo Córdova, dedicó buena parte de su obra a explicar cómo la Revolución, la nación y el Estado resultaban indisolublemente vinculados. En el prólogo de una recopilación de ensayos dedicados al presidencialismo, Córdova escribe que hay una relación "interna e indiscutible" entre el Estado del siglo xx y la Revolución. ${ }^{9}$ Una opinión que Rafael Segovia compartía en un sentido más amplio. Si el pueblo ha construido la nación, en especial en el movimiento de 1910, el conflicto social que traerá consigo cumplir los objetivos revolucionarios solamente podrá regularlo el Estado que resultó de él. La respon-

${ }^{8}$ Claudio Lomnitz, Deep Mexico, Silent Mexico: An Anthropology of Nationalism, Minneapolis, University of Minnesota, 2001; Claudio Lomnitz, Exits of Labyrinth: Culture and Ideology in the Mexican National Space, California, University of California, 1993.

9 Arnaldo Córdova, La Revolución y el Estado mexicano, México, Era, 1989, p. 11. 
sabilidad del Estado estriba en alcanzar objetivos y, además, en mantener la estabilidad política y la paz social. ${ }^{10}$

La explicación tiene una lógica sorprendente que, en especial, Arnaldo Córdova expuso en sus escritos. En un ensayo de los años setenta, cuyo título no deja dudas de su inspiración ideológica (México: revolución burguesa y política de masas) ${ }^{11}$ Córdova hace una aguda lectura de la Constitución y señala que el Estado que se creó en el siglo xx es la expresión clara de los propósitos revolucionarios. La nación, que finalmente aparece en esa época, es la única propietaria de los bienes del país porque de su correcta administración dependerá alcanzar los objetivos sociales. Pero la nación, explica Córdova, no puede hacerlo por sí misma, así que delega su representación en el Estado y concretamente en el poder ejecutivo. Por eso, la Constitución de 1917 otorga al ejecutivo la facultad de intervenir en las relaciones de propiedad (artículo 27) y arbitrar el conflicto de clases (artículo 123). Claramente aparece ahora otra asociación relevante. Si antes Lombardo había vinculado los tres episodios históricos a un solo motivo y actor social, ahora Córdova vincula la nación con el Estado y con el gobierno, regulado todo por la normatividad constitucional que, bien se sabe, fue resultado del movimiento revolucionario.

La materialización de estas relaciones históricas será el proceso de institucionalización del Estado. El mismo Córdova lo reconoce cuando afirma que la Revolución solamente puede cumplir con sus tareas si el Estado crea los medios para hacerlas realidad, medios que no son solamente programas o políticas generales (sociales y económicas), sino instituciones específicas para alcanzarlas. ${ }^{12}$ Sin estar del todo consciente del alcance de su propuesta, Córdova le dio la mejor justificación al PRI. Como se ha dicho en repetidas ocasiones, el nombre del partido entraña una contradicción conceptual

${ }^{10}$ R. Segovia, art. cit., p. 42.

${ }^{11}$ A. Córdova, op. cit., pp. 39-40.

${ }^{12}$ Loc. cit. 
que solamente se explica, como lo dice la propia Declaración de Principios en su fundación, porque se asume responsable de cumplir los propósitos de la Revolución, no mediante el conflicto de clases, sino por la acción deliberada de las instituciones que le dieron cuerpo al sistema político posrevolucionario. Así, Revolución e instituciones se vinculan, y el nacionalismo revolucionario deja de ser solamente una ideología para convertirse en un régimen político constituido por instituciones.

El Estado resulta responsable casi absoluto del bienestar de la sociedad. Como lo dijera Lázaro Cárdenas en varias ocasiones, la desigualdad y los desequilibrios de la sociedad mexicana eran tan profundos que el Estado debía regularlos y compensar la debilidad natural de las clases inferiores. En su momento, no hablaba solamente el general y político sino el jefe del ejecutivo federal. Y, una vez más, no fueron los priistas los que le dieron congruencia al planteamiento. $\mathrm{Pa}-$ blo González Casanova, en su clásico libro La democracia en México, ya advertía que el país no podía tener una democracia al estilo occidental (elecciones libres, competencia, etcétera) debido precisamente a los desequilibrios sociales que obligaban al Estado a intervenir permanentemente. Para González Casanova, los desafíos que ya enfrentaba el régimen hacia fines de los años sesenta solamente podían resolverse si el "partido gubernamental" ampliaba su representación interna para que más grupos y sectores sociales se incorporaran, de tal manera que los gobiernos que de él surgieran dieran cabal representación a sus necesidades. ${ }^{13}$

El Estado y sus instituciones debían procurar el bienestar social promoviendo el desarrollo económico. Ramón Beteta, colaborador cercano del general Cárdenas, participante destacado en el Plan Sexenal y secretario de Hacienda de Miguel Alemán, explicaba que era impensable lograr los objetivos sociales de la Revolución basándose sólo en el campo; la in-

13 Pablo González Casanova, La democracia en México, México, Era, 1975, pp. 172-173. 
dustrialización era el mejor y más efectivo medio para conseguirlo, pero no como había sucedido en las democracias occidentales, dejándolo al libre mercado, sino mediante la regulación del Estado, que determinaría los objetivos y los medios, y garantizaría los equilibrios necesarios. ${ }^{14}$ Fue así como el desarrollo económico se asoció a la rectoría del Estado, lo que por décadas sustentó las políticas económicas del país. Contra la idea generalizada de que existe una ruptura entre los propósitos económicos del cardenismo y los del alemanismo, las políticas seguidas por los gobiernos desde los años treinta simplemente muestran distintos énfasis económicos en los medios y, desde luego, en los controles sociales, pero no en los propósitos de largo plazo. Para ningún gobierno posrevolucionario el desarrollo económico podía dejarse al libre mercado; para todos ellos, la conducción del Estado era determinante. Lo que cambia radicalmente es la atención social. ${ }^{15}$

A reserva de verlo con detenimiento más adelante, es importante señalar ahora que la principal crítica que se hará al régimen priista durante los años sesenta y parte de los setenta no está dirigida a la competencia política, electoral y partidaria, sino a los incumplimientos sociales. Córdova, de nuevo, es un expositor destacado de esta crítica. Para él, las tareas de la Revolución solamente las cumplió Lázaro Cárdenas y los demás trataron de "borrar o exterminar las huellas revolucionarias del pasado histórico del Estado mexicano". ${ }^{16}$ Todos los demás rompieron o intentaron romper con los "valores ideológicos de la Revolución", y lo hicieron no porque cancelaron la competencia electoral o controlaron los

${ }^{14}$ Ramón Beteta, Pensamiento y dinámica de la Revolución mexicana, México, México Nuevo, 1951, pp. 205-253, en especial el apartado IV.

15 Leopoldo Solís, "La política económica y el nacionalismo mexicano”, en El Colegio de México, Centro de Estudios Internacionales, Lecturas de política mexicana, México, El Colegio de México, 1977, pp. 55-75; Leopoldo Solís, Planes de desarrollo económico y social en México, México, Sepsetentas, 1975.

16 A. Córdova, op. cit., p. 12. 
movimientos sociales, sino porque abandonaron las políticas que beneficiaban al pueblo mexicano.

El nacionalismo revolucionario, como ahora puede verse claro, no fue y no ha sido una simple propuesta ideológica. Es una explicación de la historia nacional, pero también es la institucionalización del papel y la responsabilidad del Estado. Ideas, pero sobre todo instituciones que, sin duda y al menos durante algunos años, dieron reales y comprobables resultados sociales. Es por esta última razón que el nacionalismo revolucionario ha sobrevivido y aunque no siempre se hace explícito, sigue siendo el principal estímulo político de la crítica a los gobiernos. Si solamente hubiera sido la ideología del PRI, el nacionalismo revolucionario habría quedado abandonado al final del siglo pasado. Como se verá más adelante, el partido lo tuvo que dejar ir cuando la tecnocracia emprendió la reforma del Estado, pero no desapareció. Por el contrario, alimentó la crítica y la oposición, ahora en la democracia.

\section{LA IZQUIERDA, DETRÁS DE LA REVOLUGIÓN}

Definir a la izquierda como opción política siempre ha sido un problema teórico y filosófico, pero como lo prueba su mismo origen (su posición en la Asamblea francesa), es menos complicado si se le rastrea históricamente. El nacimiento de la izquierda, del socialismo e incluso del Partido Comunista Mexicano, que se fundó en 1919, son antiguos; los primeros, anteriores a la propia Revolución y sus instituciones, y el tercero, contemporáneo del periodo posrevolucionario, ${ }^{17}$ pero su presencia y su influencia nunca tuvieron el éxito esperado y, quizá lo más delicado, nunca fueron capaces de distinguirse como opción política reconocible una vez que el movimiento revolucionario dio paso a las instituciones.

${ }^{17}$ Gastón García Cantú, El socialismo en México, México, Era, 1974. 
La izquierda mexicana, al menos en su expresión más destacada, que fue la comunista, adoptó plenamente el marxismo y, por ende, se asumió como organización que reconocía la lucha de clases y que buscaría la construcción de un Estado de los trabajadores, por supuesto, no por la vía democrática. Clásica y ortodoxa posición que, como consecuencia, lo hizo rechazar cualquier forma de participación electoral. $\mathrm{Al}$ menos en el plano formal, porque en los hechos la izquierda comunista, si bien nunca fue reconocida legalmente, siempre estuvo presente y tuvo una muy visible participación en más de un suceso histórico en el siglo xx. ${ }^{18}$

Dos factores determinaron su limitación política. El primero fue su expresa declaración de buscar el poder para los trabajadores que, en su origen marxista, no admitía la participación electoral. Por vocación, se mantuvo al margen de la competencia y dio el mejor pretexto político para que el sistema mexicano nunca la reconociera formalmente por considerarla subversiva y contraria a las instituciones. Su ortodoxia, que se mantuvo obsesivamente casi ochenta años, hizo que la izquierda comunista restringiera su presencia a los sindicatos y los corredores universitarios, siempre bajo el manto de un doble juego político: su supuesta clandestinidad y el evidente reconocimiento oficial de su existencia $\mathrm{y}$ actividad que, al final, la hicieron virtualmente inofensiva. Sin duda que a los costados del viejo Partido Comunista se formaron varias corrientes y organizaciones con todo tipo de variaciones socialistas o, con más generalidad, de izquierda, pero nunca consiguieron convencer a la sociedad mexicana de que eran una opción política y menos aún electoral.

La mayor tragedia de la izquierda, ortodoxa y no ortodoxa, es que sólo reconocería la realidad hasta 1978 y no por voluntad propia, sino empujada por la reforma electoral de 1977, preparada por el propio sistema, que le abrió las puertas para que abandonara la ortodoxia y avanzara hacia una

${ }^{18}$ Un libro de obligada consulta en este tema es el de Barry Carr, $L a$ izquierda mexicana a través del siglo xx, México, Era, 1996. 
opción más liberal. No es exagerado pensar que aquella reforma tuvo como principal destinatario a la izquierda socialista y, concretamente, al Partido Comunista. El sistema y el PRI, después de los movimientos estudiantiles de 1968 y 1971, que en buena medida alentaron los movimientos guerrilleros, necesitaba fortalecer la competencia electoral y, sobre todo, incorporar una auténtica opción de izquierda entre la población. La reforma, en su momento la más avanzada, creó las condiciones para que una corriente de izquierda aceptara el cambio ideológico.

En 1978, el Partido Comunista obtuvo su registro, no sin antes abandonar su clásica declaración marxista y su propósito de crear un Estado de obreros y campesinos. No sólo ingresó al medio electoral, sino que promovió la unidad de la izquierda mexicana. El proceso fue lento y accidentado y terminó en 1987, con la creación del Partido Mexicano Socialista. Renunció a llamarse comunista, pero no a ser socialista, más por orgullo que por convencimiento, porque eso le permitió competir en los comicios. Ahora era socialista... y nacionalista. La limitación de la izquierda mexicana puede comprenderse bien si se piensa que cuando aceptó competir electoralmente en 1978, ya la izquierda chilena, ocho años atrás, había alcanzado el poder por la voluntad ciudadana en las urnas y había fundado un primer e histórico gobierno socialista por la vía democrática. Ni el ejemplo sudamericano ni el cambio de orientación en el mundo socialista fueron suficientes hasta ese momento para que la izquierda mexicana se modernizara.

El segundo, y tal vez decisivo, factor que determinó el atraso de la izquierda fue su propio discurso ideológico. Al margen de la lucha de clases y el poder para los trabajadores, la izquierda asumió como objetivo la solución de las demandas sociales, la búsqueda del bienestar de la sociedad, en especial de los sectores más desprotegidos, y la función del Estado como principal actor del proceso. Compromiso social y Estado como promotor del desarrollo, exactamente las aspiraciones del nacionalismo revolucionario. La izquierda lati- 
noamericana pudo adoptar con facilidad el discurso social no sólo por el atraso económico, sino porque en ninguno de sus países hubo una revolución que enarbolara esos principios sociales. ${ }^{19}$ Los gobiernos y los partidos se mantuvieron en un esquema entre liberal y conservador que dejó libre el compromiso con el desarrollo y el bienestar. Pero en México, la Revolución creó un discurso, un Estado y un partido comprometidos con los objetivos sociales del movimiento. La izquierda tradicional simplemente se quedó al margen de la política, sin espacio ni oportunidad de expresar apropiadamente esas demandas. Sin discurso social propio y encerrada en su atrasada ortodoxia, eso limitó su alcance político. Y lo que fue peor, la colocó siempre detrás del partido del Estado. Por más que no se le reconociera legalmente y por más que se declarara anticapitalista, el Partido Comunista estuvo presente en las organizaciones posrevolucionarias. Fue parte importante de la Confederación de Trabajadores de México (CTM) y, más aún, se integró al PRM de Cárdenas convencido de que éste era la más pura expresión de los anhelos revolucionarios. ${ }^{20}$ Como lo advirtiera González Casanova, debido a la preeminencia del discurso oficial, la oposición solamente aportaba críticas, articulaba descontento y proporcionaba al gobierno los elementos necesarios para reformular políticas. En rigor, la oposición llegó a ser funcional para el propio sistema. ${ }^{21} \mathrm{El}$ resultado de esta funcionalidad fue que el discurso oficial, nacionalista y revolucionario abarcó a la misma oposición de izquierda y penetró a la sociedad, que "habla el lenguaje oficial y participa de las metas nacionales del propio Estado".

${ }^{19}$ Y la única que lo hizo fue la cubana, en medio de la Guerra Fría, y terminó siendo comunista.

${ }^{20}$ Luis Medina, Del cardenismo al avilacamachismo, Historia de la Revolución Mexicana, t. 18, México, El Colegio de México, 1978; Alicia Hernández, La mecánica cardenista, Historia de la Revolución Mexicana, t. 16, México, El Colegio de México, 1979; Luis Javier Garrido, El partido de la revolución institucionalizada, México, Siglo xxi, 1982.

${ }^{21}$ Pablo González Casanova, El Estado y los partidos políticos en México, México, Era, 1982, p. 11. 
Adicionalmente, el partido incorporó a las grandes organizaciones de obreros y campesinos, con lo que, más allá de la formalidad, se convirtió en el partido de las masas. La oposición añadió a sus pérdidas la del mismo sujeto social al que dirigía su proyecto. No sin tristeza, González Casanova concluía que el "destino de una parte importante de la oposición es el PRI".22

Pero ni el Partido Comunista ni las otras versiones socialistas fueron las únicas expresiones de la izquierda. La más importante, por su influencia en la política nacional, estuvo en el PRI. Sin ánimo de reconstruir su historia, ${ }^{23}$ es importante subrayar dos de sus elementos distintivos: fue un partido que se formó deliberadamente para fortalecer el Estado posrevolucionario y se hizo como institución que agrupara a las organizaciones sociales y los principales liderazgos políticos. Fue una organización incluyente en la que convergieron diversas posiciones e intereses que, naturalmente, se movían en un amplio espectro político. Posiciones conservadoras, moderadas y liberales convergieron en el partido y, en especial, en los gobiernos, con el compromiso compartido de sostener el sistema y, en general, sus objetivos. En el PRI, e incluso en los gobiernos, siempre existieron corrientes más comprometidas con los programas sociales y otras que, con igual aprecio por el desarrollo económico, se preocuparon más por mantener la viabilidad técnica en la política económica. La tensión fue permanente, pero los resultados favorables del modelo económico atenuaron el conflicto.

La élite posrevolucionaria siempre se consideró de izquierda, incluso claramente socialista, aunque también siempre tuvo problemas para definir qué entendía por ello. Es conocida la polémica durante el gobierno de López Mateos, el cual motivó múltiples críticas del sector privado y la derecha en general, que lo acusaron de llevar a México al so-

${ }^{22}$ Ibid., p. 129.

${ }^{23}$ Rogelio Hernández Rodríguez, Historia mínima del PRI, México, El Colegio de México, 2015. 
cialismo por la nacionalización de la industria eléctrica, el incremento en la inversión pública, el apoyo a Cuba y, por encima de todo, los libros de texto gratuitos. Las declaraciones de varios priistas (Alfonso Corona del Rosal, presidente del PRI; Manuel Moreno Sánchez, presidente del Senado, y Gustavo Díaz Ordaz, secretario de Gobernación, por mencionar a los más destacados) fueron tan confusas que el mismo presidente tuvo que intentar aclararlo. Tal aclaración no resultó la más precisa, pero igualmente revela la persistencia histórica del nacionalismo mexicano porque manifestó tener como base los objetivos sociales de la Revolución. ${ }^{24}$

Como es conocido, el dilema entre racionalidad económica y compromiso social del desarrollo llevó, en 1987, a la más profunda crisis del partido del Estado y, como se verá más adelante, a que la izquierda priista lo abandonara y se reencontrara con la izquierda más tradicional. Lo ocurrido en 1987 con la Corriente Democrática fue un hecho singular en el que la crítica al modelo económico, la reivindicación de los compromisos sociales de la Revolución y el Estado, fue elaborada por la izquierda priista, no por la izquierda socialista.

Había al menos un antecedente relevante en el que un indiscutible líder del partido oficial encabezó un movimiento que agrupó a las diversas corrientes socialistas. Lázaro Cárdenas, en su enfrentamiento con López Mateos y con motivo de las agresiones estadounidenses a Cuba, fundó el Movimiento de Liberación Nacional (MLN), que con una rapidez sorprendente reunió lo mismo al Partido Comunista que a los grupos sindicales independientes y a un sector importante del PRI. Hasta que Lázaro Cárdenas, en 1963, decidió respaldar a Gustavo Díaz Ordaz como candidato oficial y dejó a la deriva al MLN, que desaparaeció con la misma rapi-

24 "Nuestra Constitución es [...] de origen popular de izquierda [...] mi gobierno es, dentro de la Constitución, de extrema izquierda": Adolfo López Mateos, declaraciones en su gira a Guaymas, Sonora, El Universal, 1 de julio de 1960. Las intervenciones de los dirigentes se encuentran en Partido Revolucionario Institucional, op. cit, t. 7, pp. 547-566. 
dez que se había creado en cuanto el general se negó a convertirlo en una oposición activa al PRI. Si la izquierda existía y era capaz de expresar propuestas y desarrollar acciones efectivas en cuanto a las necesidades de la sociedad mexicana, se le encontraba en el PRI y en el gobierno, no en la izquierda socialista. Y de la misma manera que en los años sesenta Lázaro Cárdenas fue capaz de convocar a la unidad de la izquierda, su hijo, apoyado en su mítico apellido, lo haría en 1987.

\section{LOS RESULTADOS DEL NACIONALISMO REVOLUCIONARIO}

Los sistemas autoritarios, cuya característica principal es su pluralismo limitado, no basan su legitimidad en las elecciones porque el ejercicio del poder no depende de ellas. Los comicios son procesos más plebiscitarios que electivos, porque el sistema mantiene variados controles que impiden una real competencia que ponga en riesgo el dominio del partido dominante. Como lo apunta Linz, ${ }^{25}$ la legitimidad proviene de otros factores, no siempre políticos, que en el caso mexicano fueron el desarrollo económico y el reparto de beneficios, el cual, si bien fue desigual, proporcionó mejores condiciones sociales a la población.

Una vez que la etapa armada de la Revolución terminó, dio inicio un largo proceso de concentración del poder en que el Estado fue asumiendo responsabilidades mediante instituciones y normas jurídicas que le permitieron cumplir con el mandato de conducir plenamente el desarrollo. Aunque la creación de instituciones no cesó a lo largo del siglo $\mathrm{xx}$, fue entre mediados de los años veinte y los sesenta que el Estado mexicano fundó las más importantes en el terreno económico, político y social: el Banco de México; Nacional Financiera; la Comisión Nacional Bancaria; los bancos de

${ }^{25}$ Juan Linz, Totalitarian and authoritarian regimes, Londres y Boulder, Lynne Rienner Publishers, 2000. 
crédito agrícola y ejidal, el Banco Nacional Hipotecario, Urbano y de Obras Públicas; el Instituto Mexicano del Seguro Social; el Instituto Nacional Indigenista y Petróleos Mexicanos. Más tarde, y junto con lo anterior, el Instituto de Seguridad y Servicios Sociales de los Trabajadores del Estado, la Comisión Federal de Electricidad, así como las leyes fiscal, agraria y laboral, entre muchas otras, proporcionaron al Estado posrevolucionario los instrumentos necesarios para poner en marcha la obra social.

Los campos de la salud y la educación fueron prioritarios para los gobiernos de aquellos años. Además del Instituto de Seguridad y Servicios Sociales de los Trabajadores del Estado y del Instituto Mexicano del Seguro Social, el gobierno federal fortaleció la atención médica desde la antigua Secretaría de Salubridad, más tarde de Salud, y en 1951 construyó el imponente Centro Médico Nacional. Con las tres instituciones el gobierno federal garantizó la atención médica a trabajadores del Estado, del sector privado y a la población en general. La educación, que ha sido sin duda alguna la obra más meritoria del Estado mexicano, tendría un especial impulso desde los años veinte. Desde José Vasconcelos hasta Jaime Torres Bodet, el gobierno federal asumió la tarea de educar, instruir y preparar a la población mexicana. Garantizó la atención educativa, los maestros, los libros de texto y, además, su gratuidad. Creó escuelas en todos los niveles, financió la educación superior universitaria y técnica, y fundó los institutos para preparar profesores, primero la Escuela Nacional de Maestros (cuyo antecedente se encuentra en la de Altos Estudios, de Justo Sierra, en 1910) y más tarde la Escuela Normal Superior, ambas para formar docentes que atendieran los niveles básico y básico medio.

Pudo hacerlo porque a partir de los años cuarenta, con el país pacificado y con reglas políticas claras, puso en práctica el desarrollo económico basado en la industrialización. Circunstancias internacionales y nacionales hicieron posible que México lograra entre 1940 y 1970 uno de los crecimientos económicos más destacados en el mundo en desarrollo. 
Y lo hizo en medio de una notable estabilidad política. Como se observa en el cuadro 1, en el periodo que va de 1934 a 1982, con la única excepción de la administración cardenista, el producto interno bruto (PIB) del país creció a un promedio anual superior al $6 \%$ y entre 1958 y 1970, gracias al desarrollo estabilizador, México se acercó al $7 \%$ anual. El progreso fue posible y con él, el Estado promovió un acelerado crecimiento del capital, fortaleció el sistema bancario privado, financió importantes obras de infraestructura (carreteras, presas, refinerías, etcétera) y, a sabiendas de que ello deprimiría los salarios, se ocupó de una política social que disminuyera los riesgos del conflicto.

El modelo, sin embargo, se agotó después de casi dos décadas. Como lo habían advertido varios analistas (Raymond Vernon en un destacado lugar), ${ }^{26}$ el desarrollo estabilizador entrañaba una limitación natural en su capacidad para sostenerse a sí mismo. Las posibilidades de mantener el crecimiento dependían de fuertes inversiones que el sector privado no estaba dispuesto a hacer y que presionaban el gasto público y la capacidad de recaudación. El financiamiento desequilibrado de los ingresos públicos no sólo amenazaba al gasto social sino al propio crecimiento. El modelo mostró su rigidez hacia el final de los años sesenta y ocasionó tanto los temidos desequilibrios económicos como las protestas y movilizaciones más importantes del siglo xx, que, sintomáticamente, no fueron promovidas por trabajadores, sino por las clases medias: médicos, maestros y, sobre todo, estudiantes. ${ }^{27}$

${ }^{26}$ Raymond Vernon, El dilema del desarrollo económico de México: papeles representados por los sectores público y privado, México, Diana, 1967 (edición original en inglés de 1963).

${ }^{27}$ Enrique Cárdenas, La política económica en México, 1950-1994, México, El Colegio de México, fCE, 1996; Francisco Zapata, "Población y sociedad", en Alicia Hernández (comp.), México. Mirando hacia dentro, América Latina en la historia contemporánea, t. 4, Madrid, Fundación Mapfre-Santillana, 2012, pp. 235-269. 


\section{Cuadro 1}

PIB, tasa promedio de crecimiento anual de México por sexenios (1934-2018)

\begin{tabular}{|l|c|c|}
\hline \multicolumn{1}{|c|}{ Presidente } & Periodo & Tasa de crecimiento anual \\
\hline Lázaro Cárdenas & $1934-1940$ & 4.52 \\
\hline Manuel Ávila Camacho & $1940-1946$ & 6.15 \\
\hline Miguel Alemán Valdés & $1946-1952$ & 5.78 \\
\hline Adolfo Ruiz Cortines & $1952-1958$ & 6.42 \\
\hline Adolfo López Mateos & $1958-1964$ & 6.73 \\
\hline Gustavo Díaz Ordaz & $1964-1970$ & 6.75 \\
\hline Luis Echeverría Álvarez & $1970-1976$ & 6.16 \\
\hline José López Portillo & $1976-1982$ & 6.51 \\
\hline Miguel de la Madrid Hurtado & $1982-1988$ & 0.18 \\
\hline Carlos Salinas de Gortari & $1988-1994$ & 3.91 \\
\hline Ernesto Zedillo Ponce de León & $1994-2000$ & 3.39 \\
\hline Vicente Fox Quesada & $2000-2006$ & 2.03 \\
\hline Felipe Calderón Hinojosa & $2006-2012$ & 2.04 \\
\hline Enrique Peña Nieto & $2012-2018$ & 2.22 \\
\hline
\end{tabular}

Fuente: INEGI, series históricas.

En esas circunstancias, donde lo mismo se cuestionaba la política económica que la responsabilidad social del Estado, llegaría al poder Luis Echeverría, a cuya administración Arnaldo Córdova llamó, "sin hipérbole, la autocrítica del régimen de la Revolución”. ${ }^{28}$ La izquierda priista vería en los conflictos sociales el resultado del abandono del proyecto revolucionario y forzaría al régimen a retomarlo. De ahí que la izquierda tradicional, aún confinada a las universidades, diera su apoyo al echeverrismo. Y sin duda que el presidente y su administración respondieron al llamado. Tanto en el discurso presidencial y partidario como en la política económica, el

${ }^{28}$ A. Córdova, op. cit., p. 228. 
gobierno echeverrista retomó el nacionalismo revolucionario en su esencia: política social. Apoyó a los trabajadores y reivindicó el gasto público, lo que supuso hacer del Estado no el rector de la economía, como hasta entonces había sido, sino el principal agente del desarrollo. ${ }^{29}$

La revaloración ideológica y de las instituciones fue propuesta desde el partido. Jesús Reyes Heroles, al tomar posesión como dirigente del PRI, repitió la guía presidencial: el Estado debería conducir la economía porque su responsabilidad era promover, regular, impulsar, ordenar y suplir las "omisiones" privadas. No era, decía Reyes Heroles, un cambio circunstancial, sino el cumplimiento de los objetivos revolucionarios: nacionalistas, populares, sociales, democráticos y liberales. ${ }^{30}$

La rectificación continuaría un sexenio más, esta vez fortalecida por los ingresos petroleros. Los sexenios de Luis Echeverría y José López Portillo, como se observa en el cuadro 1, tuvieron crecimientos notables, apenas unas décimas abajo que las administraciones de López Mateos y Díaz Ordaz, pero con la enorme diferencia de que lo hicieron con un gasto deficitario, financiado con una desproporcionada deuda interna y externa. Contra lo previsto, el desarrollo no continuó y, por el contrario, el país se precipitó en recurrentes y cada vez más graves crisis económicas. El Estado y sus responsabilidades fueron seriamente cuestionados, lo mismo por quienes le habían demandado mayor intervención, que por aquellos que lo calificaron de irresponsabilidad técnica y voluntarismo político.

Como nunca antes había ocurrido, ambos gobiernos provocaron un profundo desasosiego social. A los errores económicos se sumó el enfrentamiento con el sector privado y con las clases medias, que resintieron, como ninguna otra, los

${ }^{29}$ Carlos Tello, La politica económica en México, 1970-1976, México, Siglo XXI, 1980.

${ }^{30}$ Partido Revolucionario Institucional, op. cit., t. 9, p. 318. Discurso de toma de posesión de Jesús Reyes Heroles como presidente del cEN del PRI, el 21 de febrero de 1972. 
efectos del fin del crecimiento. Los gobiernos priistas fueron criticados más que en ningún otro momento y no tanto porque el partido se mantuviera en el poder por un sistema electoral amañado, sino porque su fuente de legitimidad se había agotado. Su capacidad para generar desarrollo y beneficios sociales había concluido hacia fines de los años sesenta y durante la década siguiente no había sido capaz de encontrar una alternativa sostenible. El nacionalismo revolucionario, como ideología y como resultado material, sufrió su mayor cuestionamiento. Sus principales logros históricos -crecimiento económico y estabilidad política- encontraron sus límites en los gobiernos de Echeverría y López Portillo, y el descalabro abrió la puerta a la más profunda rectificación económica y política.

\section{NAGIONALISMO REVOLUCIONARIO Y TEGNOGRACIA}

En 1982, llegó al poder una nueva elite formada durante los años de dominio priista, pero en el sector más conservador de la administración pública, que era el financiero. Esa elite se formó durante los años del desarrollo económico y en las áreas responsables de diseñarlo y aplicarlo. Para ella, la política económica había sido manejada irresponsablemente por las dos administraciones previas, sin criterios económicos, sino políticos, y además de poner en peligro el crecimiento había anulado la posibilidad de que el Estado cumpliera con sus verdaderas responsabilidades, entre ellas, el mismo bienestar social. A juicio de esta elite, altamente especializada en economía, la intervención del Estado no resuelve ni el rumbo del desarrollo ni las limitaciones del ingreso público, ni crea mejores condiciones sociales. La intervención desmedida (como lo fue entre 1970 y 1982) agregó múltiples responsabilidades al Estado, que al final lo hicieron ineficiente. Esa política redujo las posibilidades reales de desarrollar la economía al atarla a un formal y poco responsable compromiso social. Como respondía a criterios políticos que buscaban el 
apoyo popular, a intereses de grupos y partidos, y no a la racionalidad económica, sus medidas fueron populistas. ${ }^{31}$ Para esta interpretación, el discurso en el que se sustentaron los gobiernos priistas no era nacionalista ni revolucionario, sino populista e irresponsable. Este diagnóstico, cuyo origen estaba en el manejo de la política económica, pasó de la búsqueda y elección de opciones técnicas al cuestionamiento de los fundamentos históricos y políticos de los gobiernos posrevolucionarios. No sólo la política o el modelo económico estuvo en el centro de la crítica, sino el Esatdo, su fundamento y sus responsabilidades, precisamente la base ideológica del sistema político del siglo xx mexicano.

La tecnocracia que llegó con De la Madrid no sólo se propuso corregir los errores económicos y restaurar la racionalidad, sino desmontar dicha base, a la que calificó de populista, que desde los años treinta le había dado la razón de ser y el apoyo político al PRI. Fueron los gobiernos tecnocráticos priistas los que derrumbaron el fundamento político e ideológico del régimen. Una paradoja histórica que dañaría enormemente al partido, pero no al nacionalismo revolucionario que, como se verá adelante, migró del marco orgánico del PRI para quedarse con aquel sector de izquierda que siguió, y ha seguido, convencido de su valor histórico.

De la Madrid heredó la que hasta entonces había sido la más grave y más profunda crisis económica que, además, había polarizado a la sociedad mexicana. De ser la "autocrítica de la Revolución”, Echeverría y, por extensión, López Portillo se convirtieron en los ejemplos de la arbitrariedad, los excesos personales y políticos, y la incompetencia económica. De la Madrid no vaciló en aplicar una política correctiva que buscó controlar la crisis, estabilizar la economía y sentar las bases de un futuro y posible creci-

31 Carlos Bazdresch y Santiago Levy, "Populism and Economic Policy in Mexico, 1970-1982", en Rudiger Dornbusch y Sebastian Edwards (eds.), The Macroeconomics of Populism in Latin America, Illinois, The University of Chicago Press, 1991. 
miento. Los resultados fueron catastróficos, porque las medidas agudizaron el desempleo, la caída de los ingresos, la desatención social y la ausencia del crecimiento que, como se ve en el cuadro 1, hizo que el PIB de todo el sexenio fuera menor a $1 \%$.

Pero contra toda esperanza de que la nueva elite cobrara conciencia de los costos sociales, asumió la reconversión económica como un proyecto de largo plazo porque, como lo dijera Carlos Salinas en su Primer Informe de Gobierno, ${ }^{32}$ el Estado "grande", encargado de tareas sin fin, se volvió ineficaz e incapaz de cumplir con "los reclamos sociales" de los mexicanos. Solamente un Estado que se desprendiera de obligaciones excesivas, que asumiera su verdadera responsabilidad y que atendiera la economía con criterios técnicos y racionales, sería capaz de cumplir con las demandas de la sociedad. De ahí que la racionalidad fuera prioritaria y que la atención de tales demandas se convirtiera en una consecuencia del equilibrio. Lo social era secundario.

Las explicaciones y el manejo económico de De la Madrid produjeron otra paradoja política: exhibieron al PRI como partido que producía gobiernos arbitrarios y reivindicaron el nacionalismo revolucionario sin reconocerlo abiertamente. La sucesión presidencial de 1988 originó la crisis orgánica e ideológica del priismo y obligó, como nunca antes, a separar las corrientes de izquierda del control tecnocrático.

\section{LA REBELIÓN INTERNA}

La rectificación económica que se propuso desarrollar De la Madrid fue tan profunda que no podía completarse en seis años. El proyecto requería de dos condiciones: evitar que se cambiara de rumbo en el futuro, garantizando que el sucesor fuera parte del compromiso, y desplazar a la élite política tra-

${ }^{32}$ Carlos Salinas, "Primer Informe de Gobierno", Excélsior, 2 de septiembre de 1989. 
dicional. El mismo De la Madrid lo ha reconocido sin cortapisas: Carlos Salinas fue elegido porque evitaría regresar a los "excesos económicos" de los doce años anteriores y continuaría su proyecto de desplazar "a los políticos tradicionales de los puestos de mando y decisión". ${ }^{33}$ La tarea fue cumplida sin consideraciones. Si en cuanto a la política económica el presidente mantuvo una severa disciplina garantizada, entre otras razones, por la integración de un gabinete por completo identificado con él, formado en el sector financiero del cual provenía, en el resto de posiciones políticas, en especial el PRI y las gubernaturas, De la Madrid hizo cuanto pudo para eliminar a la elite política tradicional. Cubrir la política de tecnócratas fue un propósito desarrollado con firmeza. ${ }^{34}$ Nada sorprendente fue, entonces, que al acercarse la decisión sobre la candidatura presidencial varios destacados priistas cuestionaran la continuación del proyecto económico y, obligada consecuencia, al posible candidato.

La de 1987 no era la primera escisión de la élite política. Las divisiones en que cuestionaron al partido dominante fueron frecuentes entre 1940 y 1952, con Juan Andreu Almazán, Ezequiel Padilla y Miguel Henríquez Guzmán. Los tres objetaron el procedimiento de elección, la autoridad del presidente, la fortaleza del PRM-PRI y, más aún, formaron sus propios partidos y compitieron en las elecciones. Ninguno de ellos, sin embargo, logró dañar seriamente al partido del Estado y tampoco fueron capaces de sostener a sus organizaciones, que apenas y sobrevivieron a la jornada electoral. La Corriente Democrática fue distinta. El grupo disidente empezó a formarse en 1986, aunque fue públicamente reconocido en 1987 con la salida del PRI de sus principales dirigentes. Se propuso desde el principio discutir el perfil del candidato, pero considerando, en especial, su proyecto económico. El desafío se acrecen-

${ }^{33}$ M. de la Madrid, op. cit., pp. 786, 841 y 844.

${ }^{34}$ Rogelio Hernández Rodríguez, "La división de la élite política mexicana", en Soledad Loaeza (comp.), México: auge, crisis y ajuste, Lecturas del Trimestre Económico 73, México, FCE, 1992. 
tó con los protagonistas: militantes con sólida presencia y trayectoria, como Rodolfo González Guevara y Porfirio Muñoz Ledo, y Cuauhtémoc Cárdenas, el hijo del mítico general y presidente, cuyo nombre parece sinónimo de la Revolución.

Que el proyecto económico era decisivo lo prueba el primer documento de la Corriente Democrática (Morelia, septiembre de 1986), pues apenas se menciona al candidato, pero se expone ampliamente la necesidad de reconsiderar la política económica que se había desarrollado en el gobierno que terminaba. Para los firmantes, esa política era contraria a los propósitos históricos de la Revolución que habían configurado el Estado mexicano. Sin dudarlo, el grupo disidente advertía que esos principios habían sido defendidos por el PRI desde su origen, en 1929. El cambio de modelo dejaba de ser un asunto técnico, resoluble con la simple búsqueda de alternativas económicas, para ser un cuestionamiento de los fundamentos históricos e ideológicos del Estado y las tareas gubernamentales. El segundo documento, fechado en mayo de 1987, después de la expulsión de Cuauhtémoc Cárdenas del PRI, ya demandaba claramente que el partido aceptara abrir el proceso interno para que la militancia comparara propuestas y seleccionara al candidato presidencial. ${ }^{35}$

La historia es conocida. A la salida de los dirigentes, seguida de la de muchos priistas más, se añadiría la postulación presidencial de Cárdenas y la formación del Frente Democrático Nacional (FDN) al que, si bien aparecieron grupos y partidos sin credibilidad alguna (como el Partido Popular Socialista, el Auténtico de la Revolución Mexicana, el del Frente Cardenista de Reconstrucción Nacional, etcétera), se sumó la izquierda socialista tradicional que, como había ocurrido veinte años atrás con el MLN y Lázaro Cárdenas, aceptaba subordinarse al nacionalismo revolucionario priista. Las elecciones, cuestionadas hasta ahora, si bien le dieron el triunfo al PRI y a Salinas, mostraron que la disidencia se

${ }^{35}$ Luis Javier Garrido, La ruptura. La Corriente Democrática del PRI, México, Grijalbo, 1993. 
había llevado consigo no sólo militantes y experiencia política, sino la ideología y el proyecto histórico del Estado mexicano y, de paso, del mismo partido hasta entonces dominante.

Como lo ha dicho Porfirio Muñoz Ledo, en las elecciones de 1988, los votos que casi le dieron el triunfo a Cuauhtémoc Cárdenas no partieron del tradicional abstencionismo que de pronto se convenciera de participar, sino del voto duro priista. Votaron por el hijo del general Cárdenas porque representaba a la Revolución Mexicana, no a la izquierda ni mucho menos al marxismo. ${ }^{36}$ La fortaleza del nacionalismo revolucionario se prueba con la socialización generalizada en la conciencia electoral de los ciudadanos. Sostuvo a los gobiernos priistas hasta los setenta, a pesar de que en los últimos años de esa década los excesos de Echeverría y López Portillo llevaron al país a la bancarrota. Pero fue la tecnocracia, convencida de la racionalidad económica y la conversión del Estado en un eficiente administrador, lo que ratificó su vigencia, no en la izquierda socialista sino en el priismo disidente, como bien apunta Muñoz Ledo.

\section{LAS DEUDAS DE LA ALTERNANCIA}

La reivindicación del nacionalismo revolucionario ha tenido lugar en un contexto político singular. Durante la mayor parte del siglo $\mathrm{xx}$, de pleno dominio priista, la única oposición creíble fue la derecha agrupada en el PAN. La izquierda tradicional se mantuvo fuera de las disputas electorales y el priismo ocupó el espacio de las demandas sociales. Nadie cuestionaba su compromiso y herencia revolucionaria, pero en las últimas décadas del siglo pasado el PRI dejó ir su ideología de la mano de la Corriente Democrática. Con la formación del Partido de la Revolución Democrática (PRD), en 1989, la izquierda logró su mayor aspiración, no la unidad de

${ }^{36}$ Edna Monzón de Wilki y James W. Wilki, Porfirio Muñoz Ledo. Historia oral, 1933-1988, México, Debate, 2017, pp. 821, 824-825. 
las corrientes socialistas siempre en conflicto, sino el ingreso de la izquierda priista que buscaba una nueva casa. El Partido Socialista Unificado de México (PSUM), que había invertido diez años de su vida intentando integrar a los grupos de izquierda de todo tipo, se convirtió en Partido Mexicano Socialista (PMs) en 1988 y unos meses después cedió gustoso su registro a la Corriente Democrática del PRI. Como lo hiciera en otras ocasiones, la izquierda socialista se sumó al priismo cuando comprendió que su futuro era poco prometedor.

De 1979 a 1985, época en que las diferentes versiones de la izquierda partidaria participaron en elecciones, su rendimiento fue lamentable (véase el cuadro 2). A pesar de que se presentaron cuatro partidos de izquierda, apenas alcanzaron juntos, en promedio, el $2.7 \%$ de la votación. Pocos votos que demostraban que sus posibilidades de convencer al electorado eran muy reducidas, a lo que se añadía su atávica costumbre de fragmentarse reclamando la autenticidad de sus principios.

Así habría llegado la izquierda a las elecciones presidenciales de 1988 si Cárdenas y Muñoz Ledo no hubieran creado el FDN, que permitió el ingreso prácticamente de cualquier organización política que quisiera, amparada en el renombre del candidato. Todos los participantes buscaron la sobrevivencia en la disidencia priista, pero la izquierda del PMs vio la oportunidad de apoderarse de la ventaja ideológica.

Una vez terminada la jornada electoral, con sus cuestionados resultados, Cárdenas y Muñoz Ledo se propusieron construir un nuevo partido que, sin duda alguna, era el renacimiento del priismo tradicional. El PMs, muy en su línea de sobrevivencia, le ofreció su registro y su organización. Naturalmente, abandonó su discurso socialista y adoptó, palabras más o menos, el nacionalismo revolucionario. Como lo dijera Muñoz Ledo, actor principal de esta historia, el PRD asumió "la ideología de la Revolución, del PRI" ${ }^{37}$ A esta adopción, siguió el control del partido por los líderes priistas: Cárde-

${ }^{37}$ E. Monzón de Wilki y J. W. Wilki, op. cit., p. 916. 
nas, Muñoz Ledo, López Obrador. Años después llegarían a su dirección los viejos comunistas, que si algo tenían en común con los priistas, además de la mitificación del Estado, era el reconocimiento de la disciplina del partido. ${ }^{38}$

\section{CuAdro 2}

Desempeño de los partidos de izquierda en elecciones, 1979-1985 (porcentaje de votos)

\begin{tabular}{|c|l|c|}
\hline Elecciones & \multicolumn{1}{|c|}{ Partidos de izquierda } & Porcentaje de votos \\
\hline 1979 & Comunista Mexicano (PCM) & 5.1 \\
\hline & Socialista de los Trabajadores (PST) & 2.3 \\
\hline 1982 & Socialista Unificado de México (PSUM) & 3.5 \\
\hline & Revolucionario de los Trabajadores (PRT) & 1.8 \\
\hline & Socialista de los Trabajadores (PST) & 1.5 \\
\hline 1985 & Socialista Unificado de México (PSUM) & 3.3 \\
\hline & Socialista de los Trabajadores (PST) & 3.2 \\
\hline & Mexicano de los Trabajadores (PMT) & 1.7 \\
\hline & Revolucionario de los Trabajadores (PRT) & 1.6 \\
\hline
\end{tabular}

Fuente: INE, series históricas.

$\mathrm{Su}$ accidentado proceso de institucionalización tuvo lugar en medio del cambio político del sistema mexicano hacia la democracia y, a pesar de sus tropiezos, contribuyó decisivamente a conseguirla. Más allá de las distinciones de lo que era la izquierda, el PRD se constituyó, con toda justicia, en la real opción en esta línea, más cuando los partidos que formaron el FDN desaparecieron en los años siguientes. El PRD rompió el bipartidismo que se había construido en México entre

${ }^{38}$ Víctor Hugo Martínez González, Fisiones y fusiones, divorcios y reconciliaciones: la dirigencia del Partido de la Revolución Democrática, 1989-2004, México, Plaza y Valdés, Facultad de Ciencias Políticas y Sociales (UnAm), Facultad de Contaduría y Administración (UNAM) , Facultad Latinoamericana de Ciencias Sociales, Centro de Estudios Políticos y Sociales de Monterrey, 2005. 
el PRI y la derecha panista y, por primera vez, ofreció una boleta de izquierda al electorado. No obstante, a pesar de sus esfuerzos, la derrota del PRI en el año 2000 no la consiguió la izquierda, sino la derecha panista y, más aún, encabezado por un empresario. A los líderes del PRD, además de las protestas, les correspondió ser testigos y jueces de la democracia recién alcanzada y del mayor de sus logros: la alternancia.

Como ocurrió en todos los países que transitaron a la democracia, en México el cambio político estuvo estrechamente asociado al mejoramiento de las condiciones económicas y sociales de la población. Las bondades de la democracia no se aprecian por sus valores sino por la eficiencia gubernamental que puede promover el desarrollo y, con él, la riqueza necesaria para atender las demandas sociales. Más aún en el caso de México, que desde los años ochenta se sumió en el estancamiento. Esta condición que, como ha sido señalado ampliamente, daña a la misma democracia porque la hace responsable de lo que por definición no le corresponde, en México ha sido benéfica para la izquierda unificada, porque le ha permitido reivindicar el nacionalismo revolucionario por el evidente incumplimiento en el bienestar social.

El cuadro 1 muestra con toda claridad la razón. Si la administración de De la Madrid no pudo alcanzar ni siquiera un punto de crecimiento anual del PIB, los gobiernos siguientes, incluidos los más tecnocráticos del PRI y los de la alternancia panista y priista, se han movido entre el $2.4 \%$ de Enrique Peña Nieto y el 3.9\% de Carlos Salinas. El fracaso panista es mayor, porque a ellos les correspondió la hazaña de vencer al PRI y crear las expectativas del mejoramiento político y social de la nación. Su resultado: $2 \%$ de crecimiento. Las cifras son elocuentes, pero la gráfica 1 no requiere palabras ni imaginación. El desplome de la economía es inocultable. Y lo que lo hace impactante es que el desarrollo de los años cincuenta y sesenta no lo logró una izquierda socialista, sino los gobiernos priistas que, más allá del discurso, fueron capaces de construir un modelo eficiente. Al margen de lo que pueda valorarse políticamente de la democracia, 
para la mayor parte de la población mexicana los gobiernos de alternancia han fracasado en su responsabilidad de conseguir los beneficios sociales.

\section{GRÁFICA 1}

PIB, tasa promedio de crecimiento anual de México por sexenios (1934-2018)

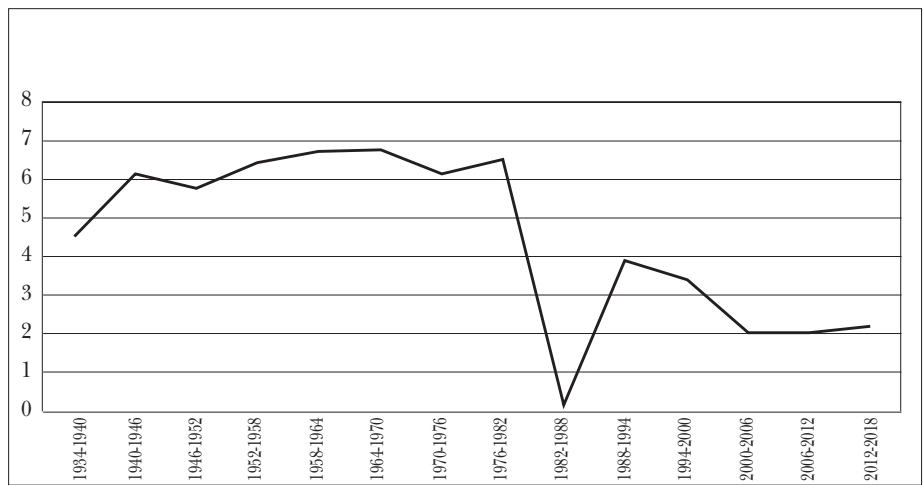

Fuente: elaboración propia con base en el cuadro 1 (arriba).

La alternancia en México, si bien se ha confirmado desde el 2000 política y partidariamente, no significó ningún cambio en la política económica. Por el contrario, con plena independencia del partido gobernante, el manejo económico estuvo en manos de la misma elite tecnocrática que aplicó la rectificación en 1982 y que tenazmente la consolidó entre 1988 y 2000. Los dos gobiernos del PAN, aunque incorporaron militantes y simpatizantes de su partido, mantuvieron al frente de la Secretaría de Hacienda y el Banco de México a reconocidos tecnócratas que profundizaron el control económico, la retracción del Estado y la desatención social.

El regreso del PRI en 2012 tampoco significó un cambio en la conducción económica. El gobierno de Peña Nieto asumió la racionalidad económica como condición de la modernidad. La política gubernamental se condujo de acuerdo 
con los mismos principios de racionalidad y control de las finanzas públicas para garantizar la estabilidad económica. El resultado fue el mismo: precario crecimiento y gasto social limitado. El PRI, en el gobierno de Peña Nieto, continuó con el abandono de su origen social e ideológico. Y, en contraste, reafirmó el nacionalismo revolucionario en la oposición que había fomentado.

Entre 2014 y 2015, el PRD sufrió una costosa escisión encabezada por el último de sus dirigentes salido del viejo PRI, Andrés Manuel López Obrador. Construyó un nuevo partido con los grupos más radicales y dispuestos a la movilización social del PRD. Su nombre, Movimiento Regeneración Nacional (Morena), recuerda a Flores Magón; su emblema recupera a Juárez; su discurso apela al pueblo desprotegido y abandonado. Jefe de gobierno del Distrito Federal con un indiscutible triunfo electoral, en la misma competencia en la que el PAN venció al PRI en el año 2000, y dos veces candidato a la presidencia de la República de esa izquierda institucionalizada, López Obrador pudo convertirse no sólo en el líder de la izquierda reciente, sino en el principal testigo y juez de los fracasos de los gobiernos de alternancia. Además de que pudo construir una crítica creíble, pudo demostrar que el PAN era tan incapaz como el PRI de mejorar las condiciones del país.

$\mathrm{Su}$ opinión tiene como fundamento la desigualdad social, el empobrecimiento de la población, el desempleo, el estancamiento de los salarios, la pérdida de calidad en la atención médica, educativa y de vivienda. En fin, en las condiciones sociales que desde los años setenta no mejoran en México. Independientemente de la violencia y la corrupción que se han agravado en los últimos gobiernos (uno panista, otro priista), la campaña de López Obrador, que dio comienzo desde la jefatura de gobierno en el año 2000, se ha centrado en la justicia social. Por estrategia política, ha pasado de proclamar el gobierno de los pobres a la equidad social y a la responsabilidad del Estado, ha reclamado la traición al nacionalismo con la reforma energética y ha prometido devolver la industria petrolera, mermada y empobrecida, al Estado. 
Como lo hiciera el PRI en el pasado, ha desempolvado la explicación de Lombardo de las tres etapas de la lucha del pueblo mexicano y se ha rodeado de los héroes nacionales más destacados: Hidalgo, Juárez, Zapata y Cárdenas. Por precaución comprensible, no ha llamado por su nombre al planteamiento que le da vida a sus propuestas: nacionalismo revolucionario. Reconocerlo sería admitir su propio origen político e ideológico. No ha ganado la izquierda tradicional, socialista, aquella del Partido Comunista y sus aliados, sino la priista, que siempre creyó en la Revolución y en la justicia social y que, más aún, con todo derecho puede decir que lo logró en el siglo pasado.

John Womack empieza su clásico libro sobre Zapata con la lapidaria sentencia de que se trata de una investigación acerca "de unos campesinos que no querían cambiar y que, por eso mismo, hicieron una revolución”. Lo único que querían era "permanecer en sus pueblos y aldeas" porque ahí habían nacido y crecido. Era lo único que conocían y querían conservarlo. ${ }^{39}$ Del mismo modo, después de un siglo de gobiernos salidos de la Revolución y de los magros resultados económicos y sociales de la alternancia democrática, la amplia mayoría de la sociedad mexicana parece estar votando nuevamente por lo único que conoce y que le garantiza beneficios sociales, aquello con lo que nació y se desarrolló. $\mathrm{Al}$ igual que el zapatismo, ha sido un cambio para preservar la tradición.

En 2018, el nacionalismo revolucionario parece cobrarse el mismo derecho que los campesinos zapatistas. Con López Obrador ha logrado la hazaña de convencer abrumadoramente al electorado mexicano de que debe reivindicarse la justicia social y el Estado comprometido con la Revolución. No ha dicho aún cómo resolverá el dilema técnico de financiar el desarrollo, que en su momento echó por tierra esa esperanza y que llevó a la crisis al país, pero no hay duda de

${ }^{39}$ John Womack, Zapata y la Revolución mexicana, México, Siglo xxI, 1984, p. XI. 
que el Estado, su política social y, por supuesto, el gasto público, volverán a ser el agente de la economía, el viejo y, a juzgar por los resultados, nada exitoso proyecto priista. Después de todo, De la Madrid parece tener razón: ¿quién puede estar en contra del nacionalismo revolucionario?

\section{REFERENCIAS BIBLIOGRÁFICAS}

Bazdresch, Carlos y Santiago Levy, "Populism and Economic Policy in Mexico, 1970-1982", en Rudiger Dornbusch y Sebastian Edwards (eds.), The Macroeconomics of Populism in Latin America, Illinois, The University of Chicago Press, 1991.

Beteta, Ramón, Pensamiento y dinámica de la Revolución Mexicana, México, México Nuevo, 1951.

Cárdenas, Enrique, La política económica en México, 1950-1994, México, El Colegio de México, FCE, 1996.

CARr, Barry, La izquierda mexicana a través del siglo Xx, México, Era, 1996.

Córdova, Arnaldo, La Revolución y el Estado mexicano, México, Era, 1989.

"Declaraciones en su gira a Guaymas, Sonora", (declaraciones de Adolfo López Mateos), El Universal, 1 de julio de 1960.

Documento de la Corriente Democrática, Morelia, septiembre de 1986.

García Cantú, Gastón, El socialismo en México, México, Era, 1974.

GARRIDO, Luis Javier, El partido de la revolución institucionalizada, México, Siglo xxi, 1982.

Garrido, Luis Javier, La ruptura. La Corriente Democrática del PRI, México, Grijalbo, 1993.

González Casanova, Pablo, El Estado y los partidos políticos en México, México, Era, 1982.

González Casanova, Pablo, La democracia en México, México, Era, 1975.

Hernández, Alicia, La mecánica cardenista, Historia de la Revolución Mexicana, t. 16, México, El Colegio de México, 1979. 
Hernández Rodríguez, Rogelio, "La división de la élite política mexicana”, en Soledad Loaeza (comp.), México: auge, crisis y ajuste, Lecturas del Trimestre Económico 73, México, FCE, 1992.

Hernández Rodríguez, Rogelio, Historia mínima del PrI, México, El Colegio de México, 2015.

Linz, Juan J., “An Authoritarian Regime: Spain”, en Eric Allardt y Stein Rokkan (eds.), Mass Politics, Nueva York, The Free Press, 1970.

Linz, Juan J., Totalitarian and authoritarian regimes, Londres y Boulder, Lynne Rienner Publishers, 2000.

Lombardo Toledano, Vicente, "Definición de la nación mexicana”, en su libro La Revolución Mexicana, 1921-1967, t. I, introducción y selección de documentos por Gastón García Cantú, México, INeHrm, 1988.

Lomnitz, Claudio, Deep Mexico, Silent Mexico: An Anthropology of Nationalism, Minneapolis, University of Minnesota, 2001.

Lomnitz, Claudio, Exits of Labyrinth: Culture and Ideology in the Mexican National Space, California, University of California, 1993.

Madrid, Miguel de la, Cambio de rumbo, México, FCE, 2014.

Martínez González, Víctor Hugo, Fisiones y fusiones, divorcios y reconciliaciones: la dirigencia del Partido de la Revolución Democrática, 1989-2004, México, Plaza y Valdés, Facultad de Ciencias Políticas y Sociales (unAM), Facultad de Contaduría y Administración (UNAM), Facultad Latinoamericana de Ciencias Sociales, Centro de Estudios Políticos y Sociales de Monterrey, 2005.

Medina, Luis, Del cardenismo al avilacamachismo, Historia de la Revolución Mexicana, t. 18, México, El Colegio de México, 1978.

Monzón de Wilki, Edna y James W. Wilki, Porfirio Muñoz Ledo. Historia oral, 1933-1988, México, Debate, 2017.

Partido Revolucionario Institucional, Historia documental del partido de la Revolución, México, ICAP, 1982, t. 5.

Salinas, Carlos, "Primer Informe de Gobierno", Excélsior, México, 2 de septiembre de 1989.

SEgovia, Rafael, "El nacionalismo mexicano. Los programas políticos revolucionarios (1929-1964)”, en El Colegio de México, Centro de Estudios Internacionales, Lecturas de política mexicana, México, El Colegio de México, 1977, pp. 39-42. 
Solís, Leopoldo, "La política económica y el nacionalismo mexicano", en El Colegio de México, Centro de Estudios Internacionales, Lecturas de política mexicana, México, El Colegio de México, 1977, pp. 55-75.

Solís, Leopoldo, Planes de desarrollo económico y social en México, México, Sepsetentas, 1975.

Tello, Carlos, La política económica en México, 1970-1976, México, Siglo Xxi, 1980.

Vernon, Raymond, El dilema del desarrollo económico de México: papeles representados por los sectores público y privado, México, Diana, 1967 (edición original en inglés de 1963).

WoмAск, John, Zapata y la Revolución mexicana, México, Siglo xxi, 1984.

Zapata, Francisco, "Población y sociedad", en Alicia Hernández (comp.), México. Mirando hacia dentro, América Latina en la historia contemporánea, t. 4, Madrid, Fundación Mapfre-Santillana, 2012, pp. 235-269. 